\title{
Comparative Analysis of Scoring Systems for Predicting Mortality in Acute Pancreatitis
}

\author{
Anurag Lavekar ${ }^{1}$, Aditi Deshpande ${ }^{2 *}$, Swarali Tadwalkar ${ }^{3}$
}

\author{
*Corresponding author: \\ Dr Aditi Deshpande \\ Flat 4, Building $10 \mathrm{D}$, SiddharthNagar \\ CHS Phase 2, Aundh, pune-411007 \\ Tel.: +6597234255/+919987799876 \\ E-mail: draditidesh@gmail.com
}

\author{
${ }^{1}$ Hepato-gastroenterologist, Ramkrishna Care Hospital, Raipur, Chhattisgarh, India \\ ${ }^{2}$ Paediatric Surgeon, Lokmanya Tilak Municipal Medical College, Mumbai, Maharashtra, India \\ ${ }^{3}$ Principal Epidemiologist, DRG, Clarivate, Mumbai, Maharashtra, India
}

Received: 14.01.2021

Accepted: 28.03.2021

Copyright (C) Celsius Publishing House www.sgo-iasgo.com

\section{ABSTRACT}

Background: In the view of need for early identification of cases of Acute Pancreatitis [AP] who may end up dying in spite of rigorous treatment measures, many scores have been proposed claiming to be superior in doing so. This study compares 5 newer scores which are possible to be assessed in first few hours of admission with mortality as outcome.

Methods: It's a retrospective study of 200 consecutive cases of first episodes of AP in a tertiary care centre in India. Scores were compared at their optimal cut off values by sensitivity, specificity, Positive and Negative predictive Values and Odds ratios.

Results: This study showed maximum sensitivity of mortality prediction by HAPS, but best PPV by CTSI. Best specificity was achievable by HAPS.

Conclusion: The newer scores have a reliable predictive capability for mortality outcome at earlier stage of management, irrespective of severity of AP.

Key words: acute pancreatitis, scores, prediction of mortality

\section{INTRODUCTION}

Acute pancreatitis [AP] is a common disease with an incidence of $5-80$ per 100,000 members of the population worldwide (1), although it exhibits wide variation in its geographic distribution and it has shown a steady increase in recent decades. Biliary tract stones and alcohol account for $80-90 \%$ cases of AP (2). Other causes include hypertriglyceridemia, hypercalcemia, post-endoscopic retrograde cholangio-pancreatography [ERCP] and drug-induced pancreatitis, but they are much less common. The clinical course of AP is usually mild and often resolves without sequelae. Nonetheless, between 10 and $20 \%$ of patients experience a severe AP attack (3), resulting in an intense inflammatory response, a variety of local and systemic complications, a prolonged hospital course, significant morbidity and mortality. In such patients, the acute inflamma- 
tory response may progress to Systemic Inflammatory Response Syndrome [SIRS], multiorgan failure, and / or pancreatic necrosis. However, the individual patient response to pancreatic injury is highly variable and often unpredictable. A mortality of approximately $1 \%$ among all AP but so high as $20 \%$ to $30 \%$ among those with severe acute pancreatitis [SAP] is documented in literature (4).

Clinical biomarkers that predict the development of these life-threatening complications are important to help guide patient triage and management. However, no simple test or group of tests have proven to perform reliably in stratifying the cases on the severity basis. Since the morbidity and mortality of AP differ markedly between mild and severe disease [mild $<5 \%$ vs severe $20-25 \%]$, it is very important to assess severity as early as possible. Multiple clinical criteria, biochemical parameters and imaging criteria have been used for this purpose.

Ranson, in 1974, proposed a score of objective parameters predicting the severity of AP, which has been modified by few [Imri, Banks,etc]. This score was proven to predict the outcome to optimal capacity, but had the drawback of requiring 48 hours to assess thus losing the critical time frame of management to improve the outcome if the score is high. In a large population study, modified Ranson's score has shown to have sensitivity of $75 \%$, specificity of $77 \%$, Positive Predictive Value [PPV] $49 \%$ and Negative Predictive value [NPV] $91 \%$ for the mortality prediction (5). In 1989, Larvin et al first proposed that Acute Physiology and Chronic Health Enquiry score [APACHE II], had 77\% prediction of outcome which was even better as $88 \%$ at 48 hours (6). APACHE ॥ had the advantages of consideration of major physiological risk factors, serial 24-hourly assessment giving better prediction and disadvantage being a lengthy and non-pancreatitisspecific score, thus important measures such as pancreatic injury and significant regional complications are missed. Many other scores, such as Simplified Acute Physiology [SAP] score, Medical Research Council [MRC], Pancreatitis Outcome Prediction [POP] Score, Japanese Severity score [JSS], Modified Glassgow Score [MGS], etc have been proposed since then, but very few have been proven as good predictors of morbidity and mortality after comparison with standard Ranson's and APACHE II.

Here, the study aimed to compare the mortality prediction capabilities of five newer scores which are possible to be assessed in first few hours of admission Bedside Index for Severity in Acute Pancreatitis [BISAP], Modified Marshall's Score [MMS], Systemic
Inflammatory Response Syndrome Score [SIRSS], Harmless Acute Pancreatitis Score [HAPS] and CT Severity Index [CTSI] [Modified Balthazar's score].

\section{METHODS}

This is a retrospective observational study in a prospectively collected data of consecutive cases of first episodes of AP from November 2019 to November 2020 in a tertiary care centre in India. Inclusion criteria was first episode of confirmed cases of AP admitted in the hospital. Cases with underlying chronic or traumatic pancreatitis, congenital malformations of pancreaticobiliary tract, malignancies of hepatopancreaticobiliary system, immunosuppression due to concurrent ailment or medication, pregnancy, known history of coagulative disorders or a recent history of myocardial infarction or cerebral infarction, treatment terminated for nonmedical reasons and incomplete data files were excluded. Five scores, viz BISAP, MMS, SIRSS, HAPS and CTSI were calculated at the time of admission in cases of AP at their first presentation. Annexure 1 shows the details of the components of scores. The cases were triaged as per revised Atlanta Classification and treated as per standard protocol of International Association of Pancreatology/ American Pancreatic Association guidelines (7).

All analyses were conducted in SAS 9.4. Bivariate relationship was assessed between categorical score variables and mortality outcome using odds ratios [OR] calculated based on Pearson's chi-squared $\left[\chi^{2}\right]$-test. By plotting Receiver-operator curves for mortality, AUC estimates at 95\% Confidence Interval for all the scores were compared. The AUC ranges were, by convention, between 1.0 [perfect separation of the 2 groups by the test] and 0.5 [no ability of the test to distinguish between the 2 groups]. The optimal cut offs were derived using Youden's Index at highest sensitivity and specificity. Furthermore, PPV and NPV for different cut offs were evaluated.

\section{RESULTS}

Among the 200 patients fulfilling inclusion criteria, 162 were males and 38 were females diagnosed with AP. Most of them [86\%] were less than 50 years of age. Aetiology of pancreatitis in our population has been depicted in table 1 . Overall mortality was reported in 24 [12\%] of the patients.

Receiver operating curves [ROC] yielded highest values of Area under Curve [AUC] for HAPS as 0.96 [0.91-1.00] followed by SIRSS 0.92 [0.86-0.97], as 
Table 1 - Aetiology of Pancreatitis

\begin{tabular}{lcc}
\hline Causes & Number of cases & Percentage \\
\hline Alcohol & 113 & 56.5 \\
\hline Biliary & 28 & 14 \\
\hline Both & 4 & 2 \\
\hline Other & 58 & 29 \\
\hline
\end{tabular}

shown in table 2 and figure 1. Least AUC values were observed for CTSI score, 0.78 [0.70-0.87]. Using chi-squared test, odds ratio and $95 \%$ confidence intervals were also estimated for all scores for predicting mortality. HAPS score yielded an odds ratio of 52.7 [12.3-225.2] while CTSI score yielded an odds ratio of 1.53 [1.26-1.85].

As shown in table 3, on comparing the sensitivity, specificity, PPV and NPV for all the scores under study, HAPS score presented a sensitivity of $91.7 \%$ and a specificity of $93.8 \%$ which was comparable to MMS, but BISAP presented with best specificity of $98.3 \%$.

Applying optimal cut off to the sample size, the number of patients with MMS $\geq 3$ was 22 [11\%], SIRSS $\geq 3$ was 42 [21\%], BISAP $\geq 3$ was 19 [9.5\%], HAPS $\geq 2$ was 33 [16.5\%] and CTSI $\geq 6$ was 89 [44.5\%]. All 17 patients with a HAPS of 3 died. However, only $25 \%$ of

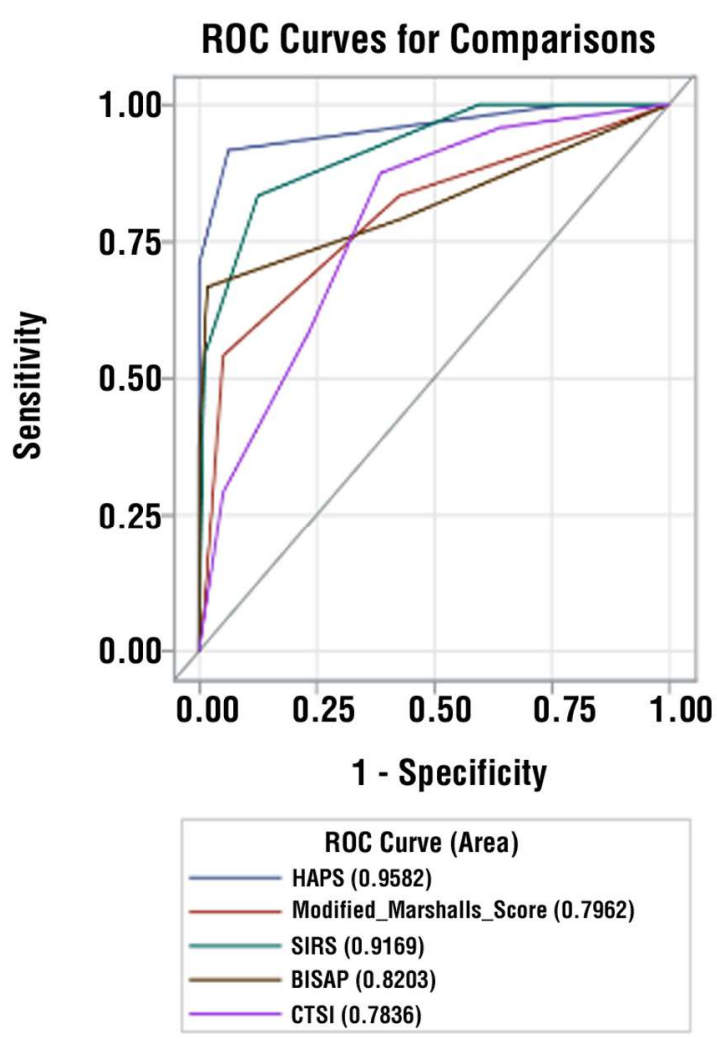

Figure 1 - Graphic presentation of ROC curves of the scores
Table 2 - Comparison of AUC and ORs of the scores

\begin{tabular}{|c|c|c|c|}
\hline \multicolumn{4}{|c|}{ AUC of different scoring systems for mortality } \\
\hline \multirow[t]{2}{*}{ Parameter } & \multirow[t]{2}{*}{ Area } & \multicolumn{2}{|c|}{ 95\% Wald Confidence intervals } \\
\hline & & Lower limit & Upper limit \\
\hline HAPS & 0.9582 & 0.9105 & 1 \\
\hline SIRSS & 0.9169 & 0.8622 & 0.9716 \\
\hline BISAP & 0.8203 & 0.7034 & 0.9373 \\
\hline MMS & 0.7962 & 0.69 & 0.9023 \\
\hline CTSI & 0.7836 & 0.6964 & 0.8709 \\
\hline \multicolumn{4}{|c|}{ OR for Mortality stratified by different scores (95\%) } \\
\hline \multirow[t]{2}{*}{ Parameter } & Odds ratio & \multicolumn{2}{|c|}{ 95\% Wald Confidence intervals } \\
\hline & & Lower limit & Upper limit \\
\hline HAPS & 52.697 & 12.33 & 225.228 \\
\hline SIRSS & 11.695 & 5.16 & 26.508 \\
\hline BISAP & 7.775 & 3.596 & 16.811 \\
\hline MMS & 4.261 & 2.344 & 7.746 \\
\hline CTSI & 1.525 & 1.259 & 1.848 \\
\hline
\end{tabular}

Table 3 - Efficiencies of the scores

\begin{tabular}{lcccc}
\hline Parameter & Sensitivity & Specificity & PPV & NPV \\
\hline HAPS & 91.7 & 93.8 & 33.3 & 1.2 \\
\hdashline SIRS & 83.3 & 87.5 & 52.4 & 2.5 \\
\hdashline BISAP & 66.7 & 98.3 & 15.8 & 4.4 \\
\hdashline MMS & 54.2 & 94.9 & 40.9 & 6.2 \\
\hdashline CTSI & 87.5 & 61.4 & 76.4 & 2.7 \\
\hline
\end{tabular}

patients had a mortality event among patients with a CTSI score $\geq 8$. For SIRSS $\geq 3,48 \%$ reported mortality while for BISAP score of $\geq 3$ had $84 \%$ of patients died. On the other hand, among patients who died, 5 patients had a BISAP of 1 . Odds of predicting mortality were the highest for HAPS followed by SIRSS.

All the statistical analyses in this study are deduced and certified by Statistician who is a co-author of the study.

\section{DISCUSSION}

In this study, the newer multifactorial scores are compared which are not yet universally accepted for morbidity and mortality prediction in AP. Though there have been discrete studies proving superiority of individual scores in this list for predicting outcome over the traditional scores with proven efficacy such as Ranson's, APACHE II or modified Glasgow Score, to the best of our knowledge, there is no literature comparing these scores, which can be assessed within few hours of admission, with each other. Keeping the null hypothesis as none of these scores had better predictability of mortality over others, results negated it after analysing the AUC and odds ratios for each score coming to 
conclusion that HAPS had the best discriminative abilities followed closely by SIRSS. The overall mortality in our sample size was $12 \%$ which was higher than a previous American study (8), but almost same as another study in a heavy intake tertiary care centre in India (9).

AP occurs when pancreatic enzymes are prematurely activated inside the pancreas leading to autodigestion of the gland and local inflammation. These enzymes can also reach the bloodstream, triggering the inflammatory cascade, which leads to the SIRS. Multiorgan dysfunction syndrome, the extent of pancreatic necrosis, infection, and sepsis are the major determinants of mortality in AP. Thus, 5 multifactorial scoring systems were selected which take these elements into consideration and as a result, are very much likely to predict the mortality.

For simplified understanding of these five scores, the summary of their components, advantages and flaws is shown in Annexure 1.

There are multiple comparative studies of these scores with traditional scores showing competitive efficacies.

BISAP has a component as SIRSS, so its efficacy parallels SIRSS. Majority of the literature has shown BISAP to have a very accurate mortality prediction (10). An Indian study performed in Uttar Pradesh with a cohort of 72 patients, had shown BISAP to have sensitivity of $100 \%$ for prediction of mortality while it was compared with APACHE II, SIRSS, CTSI and individual markers like procalcitonin, CRP, etc (9). On the other hand, our study has shown a sensitivity of $66.7 \%$ for outcome of mortality at the cut off of 3 . BISAP has been shown to be the best predictor of mortality when compared to CTSI and SIRSS in another Indian study with a sample size of 105 (11). Another recent study conducted in China has shown that BISAP and Ranson's score have comparable ability [no statistically significant difference) in predicting overall outcome [SAP, local complications, organ failure and mortality) of patients with AP (12). This study of 442 subjects has also concluded that combination of BISAP with HAPS can better help predict the prognosis of AP patients. HAPS on its own has shown a sensitivity of $98 \%$ and specificity of $77 \%$ for detecting disease severity (13). This study showed moderate agreement and positive correlation between HAPS and Ranson's score with kappa $=0.77$ at $p<0.001$.

CTSI has been primarily designed to identify the need for intervention in local complications. It, being a purely radiological score, with no clinical or biochemical /haematological component, hasn't shown comparable correlation with mortality in previous literature as good as Ranson's, APACHE II or BISAP $(9,14,8)$, but it has conclusively shown superiority in more accurately diagnosing clinically severe disease and better correlate with pancreatic infection and the need for intervention (15).

Though SIRSS and MMS have been widely used in AP management, there is a very scarce literature about their prediction abilities with mortality as outcome. SIRSS at intervals of 24 hours shows a trend in the

Annexure 1

\begin{tabular}{|c|c|c|c|}
\hline Score & Components & Strengths & Weaknesses \\
\hline MMS & $\begin{array}{l}\text { 1. PaO2/FiO2 } \\
\text { 2. Serum creatinine } \\
\text { 3. Systolic Blood pressure }\end{array}$ & $\begin{array}{l}\text { 1. Easy and early derivation possible } \\
\text { 2. Assesses Multi organ involvement }\end{array}$ & 1. Doesn't assess immune response of the body \\
\hline SIRSS & $\begin{array}{l}\text { 1. Pulse } \\
\text { 2. Respiration } \\
\text { 3. Temperature } \\
\text { 4. WBC count }\end{array}$ & $\begin{array}{l}\text { 1. Higher component of clinical factors, thus easy to assess } \\
\text { 2. Can be calculated even in clinically suspected cases, } \\
\text { even before diagnosis, within an hour of admission }\end{array}$ & $\begin{array}{l}\text { 1. Nonspecific nature of the scoring } \\
\text { 2. Comorbidities are not taken into consideration }\end{array}$ \\
\hline BISAP & $\begin{array}{l}\text { 1. Blood urea nitrogen (BUN) } \\
\text { 2. Impairment of mental status } \\
\text { 3. Systemic inflammatory response } \\
\text { syndrome (SIRS) score } \\
\text { 4. Age } \\
\text { 5. Pleural effusion }\end{array}$ & $\begin{array}{l}\text { 1. Simplicity of derivation } \\
\text { 2. Detects immune response to injury } \\
\text { 3. considers old age and dependent systemic changes }\end{array}$ & $\begin{array}{l}\text { 1. cannot easily distinguish transient organ } \\
\text { dysfunction from persistent organ dysfunction } \\
\text { at } 24 \mathrm{hrs}\end{array}$ \\
\hline HAPS & $\begin{array}{l}\text { 1. Hematocrit } \\
\text { 2. Serum creatinine } \\
\text { 3. Rebound tenderness or guarding }\end{array}$ & $\begin{array}{l}\text { 1. Only } 3 \text { factors are needed for assessment } \\
\text { 2. Involves basic laboratory tests, possible to } \\
\text { be calculated within } 30 \text { minutes of admission }\end{array}$ & $\begin{array}{l}\text { Not a good score for severe cases of AP, } \\
\text { rather useful in triaging non-severe cases of AP } \\
\text { (Talukdar et al., 2014) }\end{array}$ \\
\hline CTSI & $\begin{array}{l}\text { CT grade of pancreatitis }+ \\
\text { Necrosis score }\end{array}$ & $\begin{array}{l}\text { Directly assesses morphologic severity of necrosis } \\
\text { and inflammation }\end{array}$ & $\begin{array}{l}\text { 1. Costly investigation and CT is not indicated in very } \\
\text { mild cases of AP } \\
\text { 2. Claimed to be solely not reliable, needs one or the } \\
\text { other clinical criteria } \\
\text { 3. Based on local complications and has the drawback } \\
\text { of not reflecting the systemic inflammatory response. }\end{array}$ \\
\hline
\end{tabular}


management outcome and disease progress. Persistent organ dysfunction is correlated with persistently raised SIRSS 48 hours apart, which indirectly points towards grave outcome, thus indicative of mortality $(16,17)$. MMS is used for stratification in revised Atlanta classification in 2012 on the basis of respiratory, renal and cardiovascular systems as indicative of multiorgan dysfunction in SAP.

This study has several strengths. The first is that specific scores were selected for comparison which can be performed within the first few hours of diagnosis and admission, thus its logically intriguing to find out the best predictors in early hours. Second is exclusion of chronic pancreatitis and other comorbid conditions which had different behaviour of presentation than isolated acute pancreatitis. The limitation of all scoring systems discussed here may be that they convert continuous variables into binary values of equal weight and fail to capture multiplicative effects based on the interactions of interdependent systems. Another limitation was that the morbidity status in short or long term couldn't be evaluated and stratification of the cases based on severity in view of inadequacy of the available data in those contexts, plus the extended scoring advantage of serial assessments is not taken into consideration e.g., SIRSS at 48 hours adds up in prediction of mortality by defining persistent organ dysfunction as against transient (16).

\section{CONCLUSION}

This study showed maximum sensitivity of mortality prediction by HAPS, but best PPV by CTSI. Best specificity was achievable by HAPS. With our study and previously available literature, it can be concluded that newer scores have a reliable predictive capability for mortality outcome at earlier stage of management, irrespective of severity of AP.

\section{Conflict of interest}

All author declare that they have no conflict of interest.

\section{Ethical approval}

Institutional ethical clearance was obtained prior to the study.

\section{REFERENCES}

1. Cho JH, Kim TN, Kim SB. Comparison of clinical course and outcome of acute pancreatitis according to the two main etiologies: Alcohol and gallstone. BMC Gastroenterol. 2015;15:87.

2. Schwartz. Schwartz's Principles of Surgery 10th Ed. Publication Oral Surgery. 2007.

3. Kim BG, Noh MH, Ryu CH, Nam HS, Woo SM, Ryu SH, et al. A comparison of the BISAP score and serum procalcitonin for predicting the severity of acute pancreatitis. Korean J Intern Med. 2013;28(3):322-9

4. Venkatesh NR, Vijayakumar C, Balasubramaniyan G, Chinnakkulam Kandhasamy S, Sundaramurthi S, G. S. S, et al. Comparison of Different Scoring Systems in Predicting the Severity of Acute Pancreatitis: A Prospective Observational Study. Cureus. 2020; 12(2):e6943.

5. Forsmark CE, Baillie J. AGA Institute Technical Review on Acute Pancreatitis. Gastroenterology. 2007;132(5):2022-2044.

6. Larvin M, Mcmahon MJ. Apache-II score for assessment and monitoring of acute pancreatitis. Lancet. 1989;334(8656): 201-205.

7. Working Group IAP/APA Acute Pancreatitis Guidelines. IAP/APA evidence-based guidelines for the management of acute pancreatitis. Pancreatology. 2013;13(4 Suppl 2):e1-15.

8. Papachristou GI, Muddana V, Yadav D, Oconnell M, Sanders MK, Slivka A, et al. Comparison of BISAP, Ranson's, APACHE-II, and CTSI scores in predicting organ failure, complications, and mortality in acute pancreatitis. Am J Gastroenterol. 2010;105(2):435-41; quiz 442.

9. Khanna AK, Meher S, Prakash S, Tiwary SK, Singh U, Srivastava A, et al. Comparison of Ranson, Glasgow, MOSS, SIRS, BISAP, APACHE-II, CTSI Scores, IL-6, CRP, and procalcitonin in predicting severity, organ failure, pancreatic necrosis, and mortality in acute pancreatitis. HPB Surg. 2013;2013:367581.

10. Talukdar R, Sharma M, Deka A, Teslima S, Dev Goswami A, Goswami A, et al. Utility of the "Harmless Acute Pancreatitis Score" in predicting a non-severe course of acute pancreatitis: A pilot study in an Indian cohort. Indian J Gastroenterol. 2014;33(4):316-21.

11. Sharma V, Rana SS, Sharma RK, Kang M, Gupta R, Bhasin DK. A study of radiological scoring system evaluating extrapancreatic inflammation with conventional radiological and clinical scores in predicting outcomes in acute pancreatitis. Ann Gastroenterol. 2015;28(3):399-404.

12. Xin LI, Kang CHAO, Jiayan YAO, Bihui ZHONG, Minhu CHEN Evaluation of BISAP and HAP scoring system in predicting prognosis of acute pancreatitis. Chinese Journal of Pancreatology 2013;(3):157-161.

13. Kumar AH, Griwan MS. A comparison of APACHE II, BISAP, Ranson's score and modified CTSI in predicting the severity of acute pancreatitis based on the 2012 revised Atlanta Classification. Gastroenterol Rep (Oxf). 2018;6(2):127-131. Epub 2017 Jul 28.

14. Al-Qahtani HH, Alam MKh, Waheed M. Comparison of Harmless Acute Pancreatitis Score with Ranson's Score in Predicting the Severity of Acute Pancreatitis. J Coll Physicians Surg Pak. 2017; 27(2):75-79.

15. Bollen TL, Singh VK, Maurer R, Repas K, van Es HW, Banks PA, et al. Comparative evaluation of the modified CT severity index and CT severity index in assessing severity of acute pancreatitis. AJR Am J Roentgenol. 2011;197(2):386-92.

16. Mofidi R, Duff MD, Wigmore SJ, Madhavan KK, Garden OJ, Parks RW. Association between early systemic inflammatory response, severity of multiorgan dysfunction and death in acute pancreatitis. $\mathrm{Br}$ J Surg. 2006;93(6):738-44

17. Singh VK, Wu BU, Bollen TL, Repas K, Maurer R, Mortele KJ, et al. Early Systemic Inflammatory Response Syndrome Is Associated with Severe Acute Pancreatitis. Clin Gastroenterol Hepatol. 2009; $7(11): 1247-51$. 